\title{
MODELS OF PILOT BEHAVIOR AND THEIR USE TO EVALUATE THE STATE OF PILOT TRAINING
}

\author{
Miroslav Jirgl ${ }^{*}$ - Rudolf Jalovecky ${ }^{* *}$ - Zdenek Bradac ${ }^{*}$
}

\begin{abstract}
This article discusses the possibilities of obtaining new information related to human behavior, namely the changes or progressive development of pilots' abilities during training. The main assumption is that a pilot's ability can be evaluated based on a corresponding behavioral model whose parameters are estimated using mathematical identification procedures. The mean values of the identified parameters are obtained via statistical methods. These parameters are then monitored and their changes evaluated. In this context, the paper introduces and examines relevant mathematical models of human (pilot) behavior, the pilot-aircraft interaction, and an example of the mathematical analysis.
\end{abstract}

K e y w ords: pilot model, modeling of human behavior, identification, MATLAB, flight simulator

\section{INTRODUCTION}

Studying human behavior is a very complex scientific topic. It reaches into many other scientific fields like medicine and engineering.

If we assume that the human/pilot and his behavior are the key elements in controlling a machine, then human abilities significantly affect the whole control process. The same applies to controlling aircraft. The human/pilot's ability to control an aircraft consists of processing large amounts of information and its consequent transformation into respective movement of aircraft controls (yoke, pedals, and engine throttle lever). This information can suddenly change, its importance level can change and often it can change at an unsuitable useful signal-to-noise ratio. The ability of the human/pilot to receive and assess this incoming information is also significantly limited by the particular flight situation during which the pilot is receiving this information (quiet flight, difficult flight, acrobatic flight, etc). The biggest enemy of a pilot is time pressure, thus a lack of time to process all incoming information. This causes time delays, sometimes quite long delays between the point of the initial input signal and the appropriate pilot's response to this input signal.

Early studies about the possible representation of typical pilot regulation elements $[1,2]$ were more or less informative. Nowadays, since modern simulation systems are becoming available, these ideas have started to be studied in more detail, even though most studies are focused mainly on controlling a vehicle [3] or generally controlling artificial systems [4].

\section{MATHEMATICAL INTERPRETATION OF HUMAN BEHAVIOR}

McRuer and his colleagues were among those who initiated the idea of a mathematical description of the human response to an external stimulus in the 1980s. This scientist laid the foundations for other scientists who will be studying human behavior in the future. He was the first to suggest a connection between a machine and a human operator (see Fig. 1).

McRuer's study analyzes the basic transfer element from the automated regulation point of view. At the same time he also analyzes human behavior, the internal processes when receiving stimulus of any type - optical, audio or motion stimuli.

McRuer's studies $[1,2]$ also show the first attempt at a mathematical description of human behavior, using autopilot regulation elements. This equation can be currently understood as the basic mathematical model of pilot dynamic features.

$$
F_{(s)}=\frac{Y_{(s)}}{X_{(s)}}=K \frac{\left(T_{3} s+1\right)}{\left(T_{1} s+1\right)\left(T_{2} s+1\right)} e^{-\tau s}
$$

where

$K$ - Pilot gain represents pilot's habits for a given type of aircraft control. If the pilot takes too long to intervene or a sudden change in system amplification occurs during the regulation process, the system could become unstable.

$T_{1}$ - Lag time constant is related to the implementation of learned stereotypes and pilot routines. When a pilot repeats certain situations several times, it leads to stereotypes and learned habits. That causes the pilot to gradually eliminate his brain activity.

\footnotetext{
* Department of Control and Instrumentation, Faculty of Electrical Engineering and Communication, Brno University of Technology, Technicka 3082/12, 61600 Brno, Czech Republic, miroslav.jirgl@phd.feec.vutbr.cz ${ }^{* *}$ Faculty of Military Technology, University of Defence, Kounicova 65, 66210 Brno, Czech Republic, bradac@feec.vutbr.cz
} 

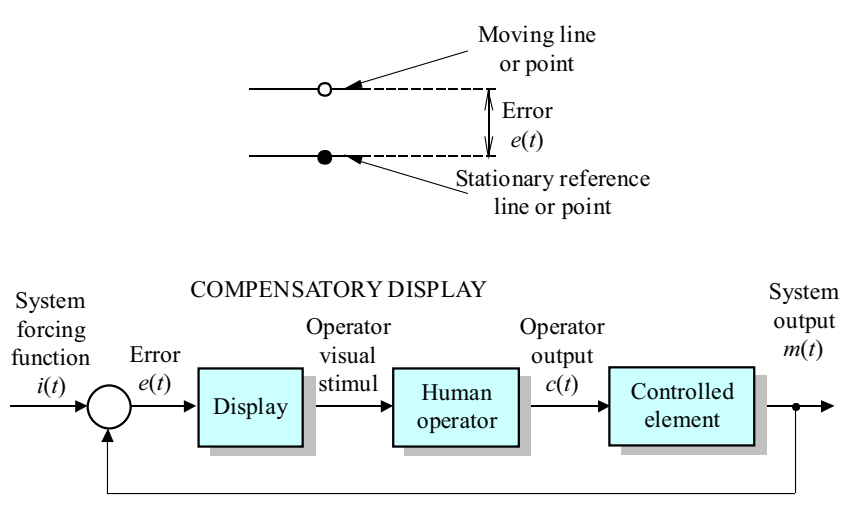

Fig. 1. Basic block diagram of manual machine control [1]

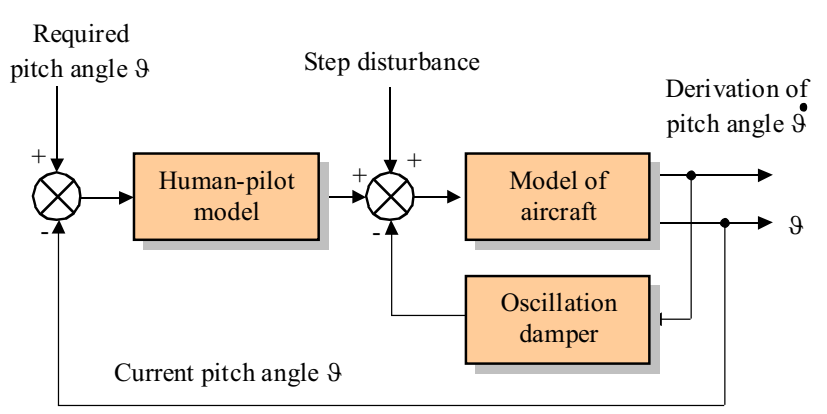

Fig. 2. Block diagram of analyzed pilot-aircraft interaction [16]
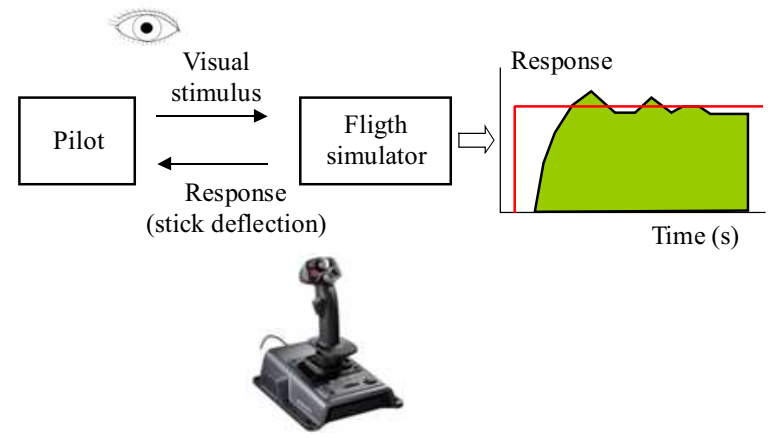

Fig. 3. The principle of testing human response to the external stimulus $[17]$

$T_{2}$ - Neuromuscular lag time constant represents the pilot's delay in his activity caused by the neuromuscular system. The neuromuscular system includes muscles and sensory organs working at a spinal level (spinal cord). The brain receives information through the spinal cord and then reacts to the external environment. The central nervous system and peripheral nervous system provide information links of the organism to the external environment and continuously regulates processes within the body.

$T_{3}$ - Lead time constant is related to the experience of the pilot. It reflects the pilot's ability to predict near future control input, i.e., to predict the situation that may occur. Estimation and prediction of the future situation is the ability to plan future steps in the surrounding circumstances. This level represents the highest level of situational awareness. The pilot has the knowledge about the state and dynamics of the individual system elements. He also has the ability not only to understand the current situation but also to predict the future situation. The pilot obtains this ability via training and experience.

The pilot obtains this ability via training and experience.

$\tau$ - This time constant indicates the delay between eye perception and the brain response via the pilot's musculoskeletal system. This transport delay depends on the current state of the neuromuscular system and also on the pilot's physical and mental condition. Fatigue may significantly increase the transport delay and the regulatory system could become unstable.

Authors [1-3] also show simplified models of human behavior, for example omitting the inertia element with T1. This model is named the Gross model of human dynamics. Human processes without any "integration" and "differentiation" of the input signal are the simplest processes of human/pilot behavior. The pilot "only" carries out a function of an amplification element with dynamics of the power element. Time constants $T_{1}$ and $T_{3}$ in equation (1) are omitted. That makes up the simplest transfer function of human/pilot dynamics [2]. A more detailed analysis and description of the acquired features for basic human behavior models is described in [5].

Our real life experience, however, points out the unsuitability of simplifying the models. Mathematical analysis in [6] showed that for a more precise assessment of human behavior while flying an aircraft it is necessary to extend (1) by time constants $T_{4}$ and $T_{5}$. By doing that we get a transfer function of a higher order and after implementing suitable identification methods for identifying transfer function parameters we get better results. So far, authors of this article have not managed to interpret physiological or psychological processes taking place in the human body that would enable us to determine the significance of the new time constants. The required physiological interpretation of the human body is still missing, even though McRuer mentions in [1] the possibility of transfer functions of higher orders. He interprets them, however, as dampened glimpses of muscles. Other works also present the possibility of neuromuscular system extension and nonlinearities addition [7-10].

\section{APPROACHES TO PILOT-AIRCRAFT INTERACTION}

A possible connection between mathematical models of human behavior and selected models of aircraft motion in space is shown as a block diagram in Fig. $2[11,12]$. The block diagram consists of three subsystems that can vary in complexity. The human model is based on (1) that can be simplified or extended by omitting or adding time constants in inertia elements $[13,14]$. The aircraft flight model can also vary in complexity [15]. Flight is 
usually divided into the so-called longitudinal motion and side motion and the human behavior model is adjusted to this division. The third block is usually a classic damper of fast oscillations that is currently fitted to all modern aircrafts. The third block complexity, or simplicity, can be mathematically described perhaps by a linear element with amplification smaller than 1 and represents the first negative feedback. The purpose of this feedback is to lower the so-called fast oscillations along the aircraft oscillation (rotation) axis, as the pilot is unable to response quickly to these oscillations due to his delayed response to stimuli - in (1) it is called a transport delay. If the oscillation damper were not fitted to the aircraft, the pilot could increase the aircraft oscillations by his delayed response rather than reducing them.

The second feedback is the connection between the pilot and aircraft and is run via the human behavior model. Two significant inputs into the analyzed system are mechanical failures, thus undesired influences on the controlled flight parameter, and the flight program for the pilot, ie a desired change of the controlled flight parameter, provided by the pilot.

In order to acquire time constant values in (1) it is necessary to test/measure the pilot's responses to a pre-defined aircraft motion and then run the acquired responses through suitable mathematical algorithms identifications. Since we acquired a sufficient amount of pilot responses, the resultant coefficients can be then put into our pre-set simulation diagrams. Measurement of a sufficient number of pilots realized on the flight simulators at the same or very similar conditions enables the use of the acquired numerical parameters for preset simulation schemes.

It is not possible to acquire the required responses during a real flight. It is not only technically difficult but also very uneconomical. Therefore, pilot behavior responses are measured for a pre-defined flight section on a flight simulator. These simulators are suitable for preparing many standard flight situations that can be repeated under practically identical initial conditions. That allows measuring the pilot behavior and responses for many different pilots and, in addition, it also allows testing individual pilots in the course of their training.

The initial idea to measure the pilot's response to a suitable stimulus that would at least partially represent the flight control was born a few years ago and can be described by the schematic diagram in Fig. 3. The tested subject (human, pilot) is watching an external visual stimulus on a computer screen. This stimulus is a line with a sudden step change and the pilot is trying to follow this step change by moving a "point" on the screen using his yoke. This sudden step change is pre-set to a random time. The tested pilot does not know if the step change will go to positive or negative values. Thus, the human/pilot has to respond to an unexpected situation. The purpose of the test is to copy the step change as quickly and accurately as possible. The yoke movement in time is recorded by sensors in two mutually perpendicular axes, transferred into a computer and recorded together with the speed and external stimulus size. All of this information, including the time axis, is then utilized in mathematical methods of experimental identification. The calculated parameters of the human behavior models do not fully represent the pilot's load during a real flight, however, a certain realistic description of transfer function parameters for human behavior while flying an aircraft has been achieved.

\section{OPTIONS FOR MATHEMATICAL ANALYSIS OF HUMAN BEHAVIOR WHILE FLYING AN AIRCRAFT}

The scale of analysis for human behavior while flying an aircraft can be defined using a mind map, see Fig. 3. The simulator outputs are missions - selected parts of the test for a certain pilot. Before the actual mathematical analysis, an analysis method has to be selected (currently there are two analytical methods available) and the pilot behavior model has to be selected. The model is selected according to the basic (1) — ' $\mathrm{A}$ ' type pilot. ' $\mathrm{B}$ ' and ' $\mathrm{C}$ ' type pilots are simplified equations and ' $\mathrm{D}$ ' to ' $\mathrm{F}$ ' type pilots are extended equations, see Fig. 4 . The actual analysis outputs are time constants, transport delay or model linear element gain, statistical data and mainly graphical results providing clear information about the analysis. The graphs are showing the time flow of identification, root distribution, the so-called characteristic equation and transfer function of the human/pilot model.

Quite a simple simulator was built on our site using the X-Plane-10 program environment. This simulator is nowadays considered as a complex, comprehensive and highly effective flight simulator for PCs, offering advanced flight models. It is also an engineering tool for easy flight model estimates for all aircraft categories, including construction solutions. The data acquired from these mathematical-physical calculations in real time are extremely precise, with minimum deviation from the real pre-set situation. The basic version of the X-Plane software comprises 30 aircraft dynamics. It is possible to try out, for example, a Bell 206 JetRanger helicopter, a Cessna 172 aircraft, a King-Air C90, a shuttle, or a B-2 bomber. The King-Air C90 Aircraft was selected for testing the pilot's response to an unpredicted situation occurring during a flight. The simulator also enables the recording of the flight course (selected parameters) into a text file with a frequency of $20 \mathrm{~Hz}$.

The initial thought of measuring pilot responses became real on this simulator. A flight mode was defined: altitude $2900 \mathrm{ft}$, speed $170 \mathrm{mph}$, angle of attack and pitch angle, including their change, was approx. 0 (note $1 \mathrm{ft}=0.305 \mathrm{~m}$ and $\left.1 \mathrm{mph}=0.447 \mathrm{~m} \mathrm{~s}^{-1}\right)$. At a certain time the altitude was step-changed to $2600 \mathrm{ft}$ and the task of the pilot was to correct the altitude back to the original $2900 \mathrm{ft}$. A total of 8 trainee pilots were tested in this flight mode, all of them had about 40 to 50 flight hours of experience in the Zlin Z141 aircraft. Each pilot 


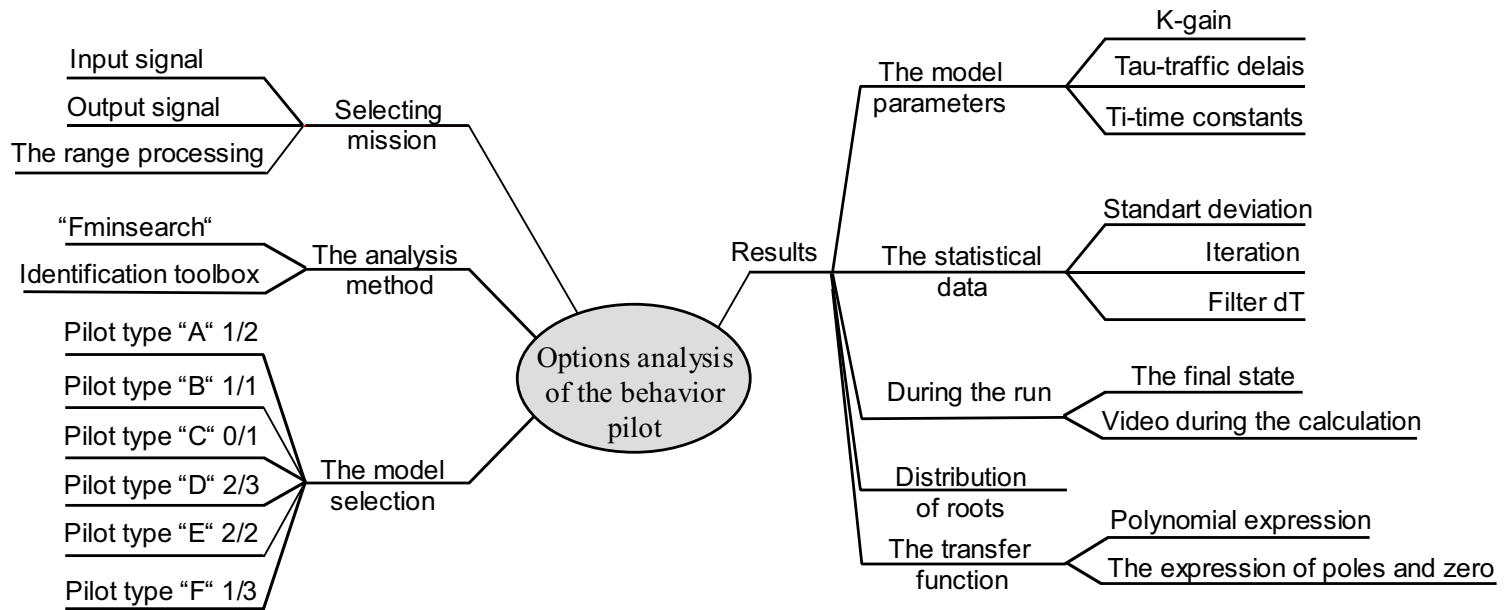

Fig. 4. Options for Mathematical Analysis of Human behavior [18, 19]
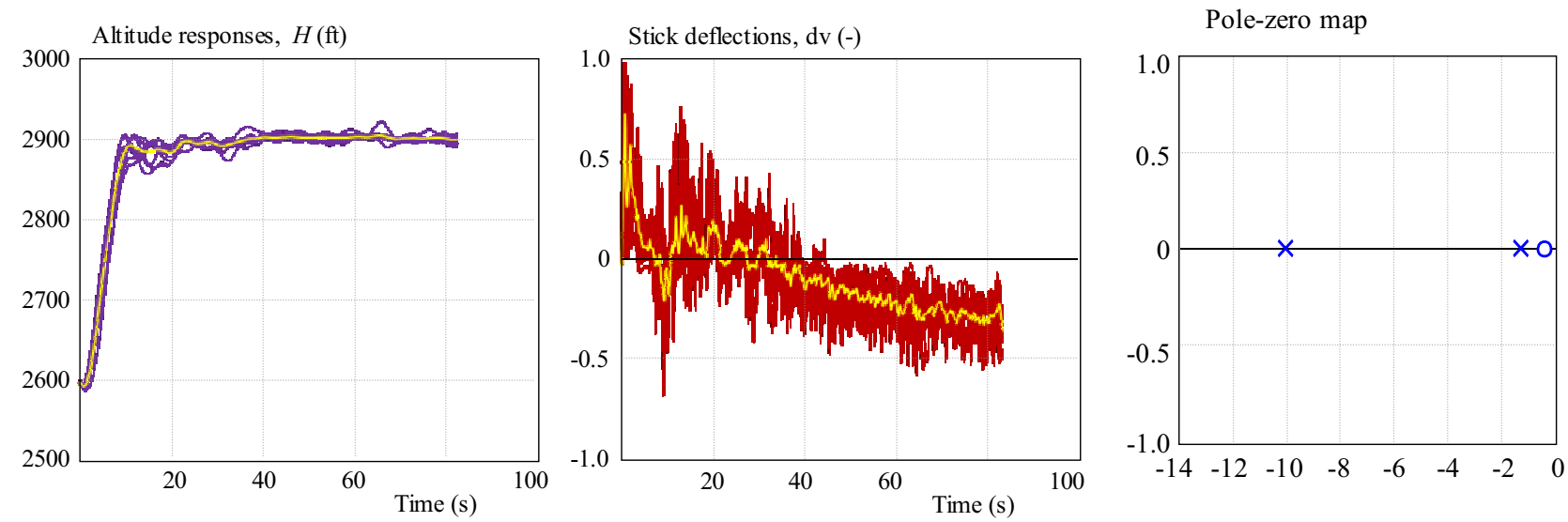

Fig. 5. Pilot's 4 data measured by flight simulator and their interpretation (see further explanation) by pole-zero map: poles at -10 , and -0.794 , zero at $-0.279 \mathrm{rad} / \mathrm{s}$
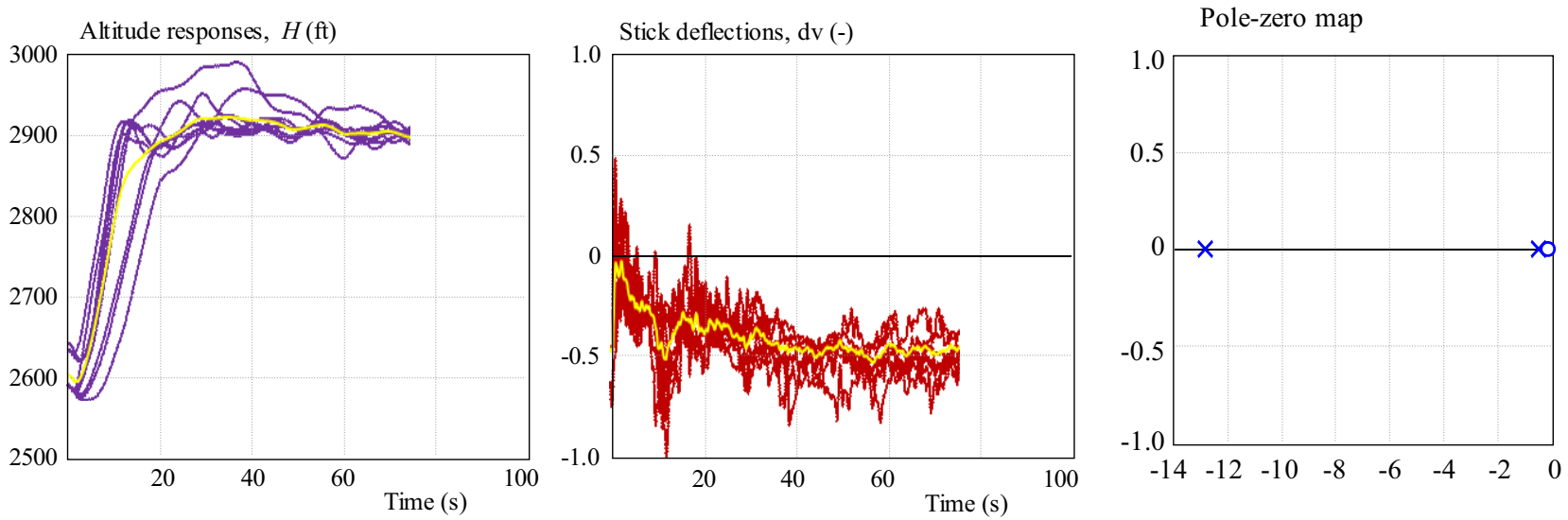

Fig. 6. Pilot's 5 data measured by flight simulator and their interpretation (see further explanation) by pole-zero map: poles at -12.5 , and -0.581 , zero at $-0.29 \mathrm{rad} / \mathrm{s}$

had the altitude changed 10 times one after another, always after putting the aircraft back into the initial flight state.

The data acquired from these tests were then run through analysis using the powerful MATLAB® simulation tool.
Two tests with two pilots (pilots No. 4 and No. 5) are presented in this article. The data acquired from the simulator, representing individual tests, are depicted in Fig. 5 and Fig. 6. These figures show individual pilot's responses (yoke deflections $\mathrm{d} v(t)$ ) to a step-change in the aircraft's altitude from $2900 \mathrm{ft}$ to $2600 \mathrm{ft}$ (left). The figures also show the evolution of the real aircraft altitude 

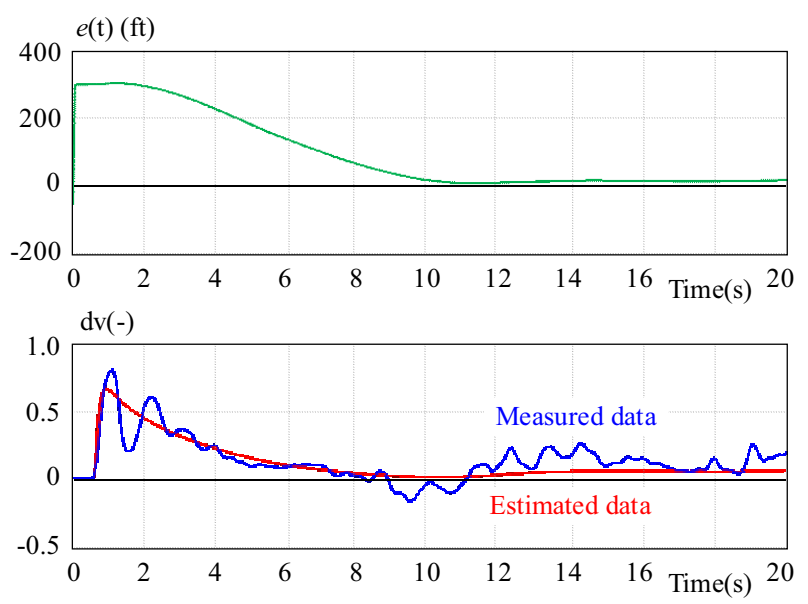

Fig. 7. The input and output data and response of identified transfer function (Pilot no. 4)

Table 1. The identified transfer function parameters

\begin{tabular}{cccccc}
\hline pilot & $K(-)$ & $T_{1}(\mathrm{~s})$ & $T_{2}(\mathrm{~s})$ & $T_{3}(\mathrm{~s})$ & $\tau(\mathrm{s})$ \\
\hline Pilot no.4 & $7.24 \times 10^{-4}$ & 0.10 & 1.26 & 3.58 & 0.55 \\
Pilot no.5 & $4.88 \times 10^{-4}$ & 0.08 & 1.72 & 3.45 & 0.95 \\
\hline
\end{tabular}

$H$. The highlighted curves are the arithmetic mean of the acquired readings.

The figures also show that individual pilots reacted differently to the altitude change. Pilot 4 responded very similarly in all of his tests. Pilot 5 was learning with each test and his responses significantly improved with each test. These tests, in addition, provide a clear overview of the adaptation ability of individual pilots to control dynamics.

By using (1) describing the dynamic pilot behavior it is possible to express the average pilot response. To identify average parameters of the transfer function the input data will be the arithmetic average of 10 test readings, responses. To describe the pilot's transfer function as a regulator of an aircraft system (a regulated system), see Fig. 2, it is necessary to express the input signal for the pilot as a control error $e(t)=i(t)-m(t)$. In this case it is the difference between the required altitude $(2900 \mathrm{ft})$ and the actual altitude.

The prepared input data was then run through the identification algorithm in MATLAB®. This algorithm used the fminsearch function and the criteria function is calculated as a sum of deviations squared

$$
K R I T=\sum_{i=1}^{n}\left(c(i)-c_{m}(i)\right)^{2} .
$$

Here $c$ is the real value of the output variable, $c_{m}$ is the modelled (estimated) value of the output variable and $n$ is the data length.

The identification process is an approximation of the initial function using responses of continual transfer in the form of (1) with parameters identified by the above
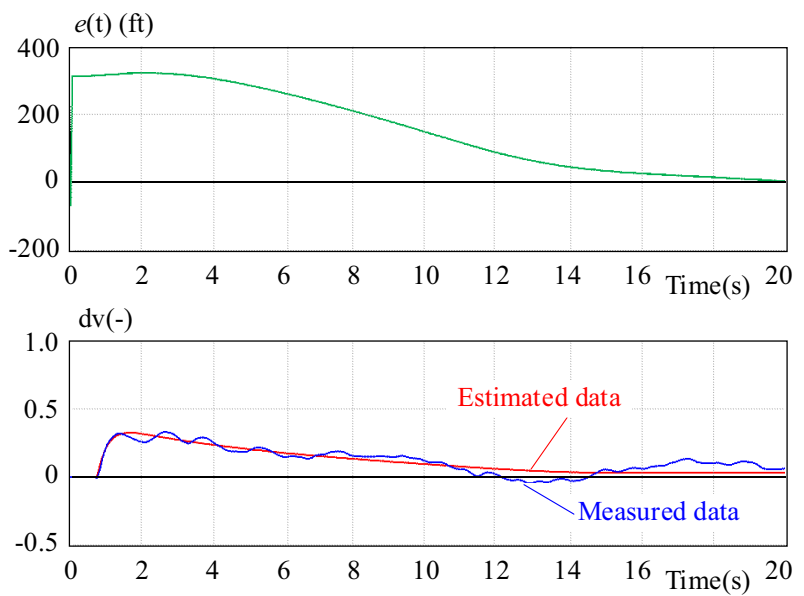

Fig. 8. The input and output data and response of identified transfer function (Pilot no. 5)

described algorithm. Input and output data, including interpolated identified transfer functions for individual pilots, are shown in Figs. 7 and 8.

The identified parameters of transfer functions describing the dynamic behavior of individual pilots are summarized in Tab. 1. These parameters are in a range stated in publications $[1,3$ and 6$]$ and their differences represent certain differences in the dynamic behavior of individual pilots. Gain $K$ depends on the input and output signal ratio and is also connected with the speed of the control process. The value of gain $K$ for pilot 4 is higher by about $30 \%$ than for pilot 5 . This difference is also clear from the measured readings. The neuromuscular constant $T_{1}$ of both pilots is in the order of $0.1 \mathrm{~s}$, which is a typical constant value for a human. The ratio of $T_{3}$ and $T_{2}$ constants shows a certain control strategy and is also connected to the pilots' experience and their ability to adapt to the control dynamics. The mean value of both pilots' response delay also varies quite a lot. The pilots' response delay is defined as the time between registering the change and transferring the information from the pilot eye and brain for processing.

Another interpretation of the measured and identified values can be a pole-zero map of the identified system, as shown right in Fig. 5, and Fig. 6.

\section{CONCLUSION}

The aim of this paper was to describe a basic model of pilots' behavior and its application in approximating real control actions. First of all, the authors characterize the employed mathematical model. For the given purpose, McRuer's linear dynamic model (the most popular instrument in this field) is used to approximate the human behavior. The following and closely related part of the article defines the approach to the aircraft-pilot interaction, which provides the necessary framework for any use of the applied procedure of pilot training evaluation. The last section of the paper presents the measurement 
and evaluation of the pilot responses carried out with two test subjects. We utilized the flight simulator at the Brno University of Defense, where approximately 80 identical cycles were run with 8 pilots. We then subjected the acquired data to an identification algorithm. The obtained results are the model parameters, thus the average time constants and the pilot response delay. These parameters describe a pilot's control ability and the dynamics of his neuromuscular system. The results confirm the theoretical assumptions described in this text and correspond to the ranges specified in other publications $[1,2,6,7,20]$.

Planned research activities in the described context include testing the same pilots in different training phases under identical conditions. Significantly, there is a possibility of using various types of simulators. We expect that after statistical evaluation of the applied analyses the mean values of the time constants will be generalized and based on the given results the state of pilot training will be evaluated.

The paper was written within the development organization project UO-K206 titled "Complex Electronic System for UAS" and a project of the Technology Agency of the Czech Republic n.TA04031376 Research/development methodology training aviation specialists L410UVP E20.

This paper was also made possible by grant No. FEKTS-14-2429 "The research of new control methods, measurement procedures and intelligent instruments in automation", and the related financial assistance was provided from the internal science fund of the Brno University of Technology.

\section{REFERENCES}

[1] McRUER, D. T.-KRENDEL, E. S. : Mathematical Models of Human Pilot Behavior, AGARD AG-188, 1974.

[2] McRUER, D. T.-KRENDEL, E. S. : Human Pilot Dynamics in Compensatory Systems, USA: Air Force Flight Dynamics Laboratory, Research and Technology Division, Air Force Systems Command, United States Air Force, 1965.

[3] HAVLIKOVA, M. : Diagnostic of Systems with a Human Operator, PhD thesis, Department of Control and Instrumentation, Faculty of Electrical Engineering and Communication, Brno University of Technology, Brno, 2008.

[4] GLODEK, M.-HONOLD, F.-GEIER, T.-KRELL, G.-NOTHDURFT, F.-REUTER, S. et al : Fusion Paradigms in Cognitive Technical Systems for Human-Computer Interaction, Neurocomputing 161 (2015), 17-37.

[5] JALOVECKY, R. : Man in the Aircraft Flight Control System, Advances in Military Technology 4 (2009), 49-57.

[6] BORIL, J.: Analysis of Pilot-Aircraft Mechatronic System in View of Automatic Control Systems, PhD thesis, University of Defence, Brno, 2005.

[7] HAVLIKOVA, M.-SEDIVA, S.-BRADAC, Z.-JIRGL, M. : A Man as the Regulator in Man-Machine Systems, Advances in Electrical and Electronic Engineering 12 (2014), 469-475.

[8] HESS, R. A.: Modeling Pilot Control Behavior with Sudden Changes in Vehicle Dynamics, Journal of Aircraft 46 (2009), 1584-1592.
[9] INNOCENTI, M.-BALluChI, A.-BALESTRINO, A. : Modeling of Nonlinear Human Operator in the Control Loop: Preliminary Results, Journal of Guidance, Control, and Dynamics 23 (2000), 736-739.

10] HESS, R. A.: Pilot Control, Principles and Practice of Aviation Psychology, Erlbaum, Mahwah, NJ (P. S. Tang and M. A.Vidulich, eds.), 2002.

[11] LONE, M.-COOKE, A. K.: Development of a Pilot Model Suitable for the Simulation of Large Aircraft, in: 27th International Congress of the Aeronautical Sciences, Cancun, 2010.

12] POOL, D. M.-ZAAL, P. M. T.-DAMVELD, H. J.—van PAASSEN, M. M.-MULDER, M. : Pilot Equalization in Manual Control of Aircraft Dynamics, Proceedings of the 2009 IEEE International Conference on Systems, Man, and Cybernetics, San Antonio, 2009, pp. 2480-2485.

[13] HESS, R. A.-MARCHESI, F. : Analytical Assessment of Flight Simulator Fidelity Using Pilot Models, Journal of Guidance, Control and Dynamics 32 (2009), 760-770.

14] HESS, R. A.-SIWAKOSIT, W.: Assessment of Flight Simulator Fidelity in Multiaxis Tasks Including Visual Cue Quality, Journal of Aircraft 38 (2001), 607-614.

[15] JIPP, M.-TEEGEN, U.-HAZAMA, K.-SAWARAGI, T. : Aircraft States and their Impact on Pilot Gaze Behavior, in: IEEE International Conference on Systems, Man, and Cybernetics, Anchorage, AK, USA, 2011, pp. 2076-2081.

[16] BORIL, J.-JALOVECKY, R.-RASHID, A. : Human - Machine Interaction and Simulation Models Used in Aviation, in: Proceedings of 15th International Conference on Mechatronics - Mechatronika, Prague, 2012, pp. 314-317.

17] JALOVECKY, R.-JANU, P.—BORIL, J. : Experimental Parameters Identification of the Human-Pilot Model Behaviour, Cybernetic Letters 1 (2016).

[18] JALOVECKY, R.: The HUFA_V Program for Archiving Acquired and Analysed Data from Human Behaviour Responses while Flying a Plane, in: Proceedings of the 14th International Conference on Measurement of Diagnostic Reliability of Aircraft on Board Systems, Brno, 2014, pp. 114-121.

[19] JALOVECKY, R.: The HUFA_M Program for Analysing Acquired Data from Human Behaviour Responses while Flying a Plane, in: Proceedings of the 14th International Conference on Measurement of Diagnostic Reliability of Aircraft on Board Systems, Brno, 2014, pp. 122-128.

[20] LONE, M.-COOKE, A. K.: Pilot-Model-in-the-Loop Simulation Environment to Study Large Aircraft Dynamics, in: Proceedings of the Institution of Mechanical Engineers Part G Journal of Aerospace Engineering 227 (2012), 555-568.

Received 9 February 2016

Miroslav Jirgl was born in 1988. He received his MSc in electrical engineering from the Brno University of Technology in 2012. Currently he is a PhD student at the Department of Control and Instrumentation, Faculty of Electrical Engineering and Communication, Brno University of Technology and his research interests include man-machine systems, human behavior modeling and flight modeling.

Rudlof Jalovecký was born in 1956. His research interests include automatic flight control, digital control systems, database systems, man-machine interactions and human behavior modelling. He is professor at the Brno University of Defence.

Zdeněk Bradáč was born in 1973. He received his $\mathrm{PhD}$ in technical cybernetics from the Brno University of Technology in 2004. His research interests include HMI systems, faulttolerant systems, information systems safety and security. He is an associated professor at the Department of Control and Instrumentation, Faculty of Electrical Engineering and Communication, Brno University of Technology. 